\title{
CESA/VASA: Tratamento da Incontinência Urinária de Urgência Idiopática por Via Laparoscópica
}

\section{CESA/VASA: Treatment of Idiopathic Urgency Urinary Incontinence by Laparoscopic Approach}

Pedro Galego ${ }^{1}$, Frederico Ferronha ${ }^{1}$, Fortunato Barros ${ }^{1}$, João Pina ${ }^{1}$

\section{RESUMO}

CESANASA é uma nova técnica que tem como objetivo principal o tratamento curativo da incontinência urinária de urgência, patologia conhecida como bexiga hiperativa idiopática. A técnica consiste numa abordagem cirúrgica abdominal (aberta ou laparoscópica) com a aplicação de uma fita (CESANASA) que se fixa ao colo uterino ou à cúpula vaginal, sendo inserida no lugar onde existiam os ligamentos útero-sagrados (ligamentoplastia), e fixada ao osso sacro. Isto faz com que, graças à tensão e suporte desenvolvidos pela fita aplicada, a bexiga volte à sua posição anatómica habitual. Este caso retrata uma doente, 72 anos, com queixas de incontinência urinária mista. Efetuou vários tipos de medicação (anti-muscarínicos e mirabegron) sem grandes melhorias. Realizou estudo urodinâmico que confirma a presença de incontinência urinária de esforço e bexiga hiperativa. Foi submetida a colocação de fita CESA por via laparoscópica + sling sub-uretral (TOT) por via transvaginal. Cirurgia e pós-operatório sem intercorrências. Após quatro semanas da intervenção, a doente foi reavaliada afirmando estar assintomática.

PALAVRAS-CHAVE: Bexiga Urinária Hiperativa; Incontinência Urinária; Procedimentos Cirúrgicos Urológicos 


\section{ABSTRACT}

CESANASA is a new technique whose main objective is the curative treatment of urgency urinary incontinence, a pathology known as idiopathic overactive bladder. The technique consists of an abdominal surgical approach (open or laparoscopic) with the application of a tape (CESANASA) that attaches to the uterine cervix or the vaginal dome, being inserted in the place where the uterosacral ligaments existed (ligamentoplasty), and fixed to the sacred bone. This makes the bladder return to its normal anatomical position thanks to the tension and support developed by the applied tape. This case reports a woman, 72-year-old, with complaints of mixed urinary incontinence. She took several types of medication (anti-muscarinic and mirabegron) without major improvements. She underwent an urodynamic study that confirmed the presence of stress incontinence and overactive bladder. She was submitted to the placement of the CESA tape by Laparoscopic + TOT by transvaginal approach. Surgery and post-operative without complications. Four weeks after surgery, the patient was re-evaluated, and she affirmed to be asymptomatic.

KEYWORDS: Urinary Bladder, Overactive; Urinary Incontinence; Urologic Surgical Procedures

\section{INTRODUÇÃO}

Esta nova técnica tem como objetivo principal o tratamento curativo da incontinência urinária de urgência, patologia conhecida como bexiga hiperativa de causa idiopática, que afeta cerca de $40 \%$ das mulheres com mais de 60 anos de idade, e um número indeterminado nas mais jovens. ${ }^{1,2}$

Os músculos e ligamentos do pavimento pélvico são responsáveis pelo suporte e controlo adequado da bexiga e dos restantes órgãos pélvicos. Segundo o "criador" da técnica, Dr. Wolfram Jäger (Diretor da Uroginecologia e Cirurgia do Pavimento Pélvico do Hospital Universitário de Colonia-Alemanha), a incontinência urinária de urgência resulta da rotura ou lassidão dos ligamentos útero-sagrados. ${ }^{1}$ Estes ligamentos, que se estendem do útero ao osso sacro e que se encarregam de dar elasticidade e força a essa região anatómica, perdem a sua capacidade de suporte e sustentação, levando a que o útero, a bexiga e o reto sofram prolapso sobre o pavimento pélvico, perdendo a sua posição anatómica original. 1,3-11

A bexiga torna-se instável e desencadeia a perda de urina por urgência, ou seja, desenvolve o aparecimento de contrações involuntárias que originam urgência miccional, polaquiúria e noctúria. Muitas vezes a doente não consegue atrasar a vontade de urinar e acaba por desenvolver urge-incontinência. São estas características que tornam esta incontinência diferente da incontinência de esforço, caracterizada por perdas de urina somente associadas a um esforço como tossir ou espirrar., 1,-11

A técnica está indicada para todas as mulheres que apresentam sintomas de incontinência urinária de urgência ou mista em que todos os tratamentos "conservadores"/ minimamente invasivos/médicos não se revelaram particularmente eficazes. ${ }^{1,3-11}$
Devem ser excluídas todas as doentes em que os sintomas sejam causados por patologias neurogénicas e congénitas. As doentes candidatas a esta técnica têm, portanto, como diagnóstico definitivo uma bexiga hiperativa de causa desconhecida (idiopática). A bexiga hiperativa idiopática corresponde a cerca de $80 \%$ dos casos de incontinência urinária de urgência. ${ }^{1-11}$

\section{CASO CLÍNICO}

Doente do sexo feminino, com 72 anos de idade, no dia 13 de fevereiro de 2015 recorreu, por queixas de incontinência urinária mista, à consulta de Urologia. Afirmava a presença de perda de urina aos esforços e por urge-incontinência. A utente manifestava perda de urina a qualquer tipo de esforço (sempre e sem qualquer controle), assim como queixas de imperiosidade vesical que originavam perda de urina (urge-incontinência). Afirmava bom jato urinário e com fluxo contínuo, esvaziamento vesical completo e que evacuava sem dificuldades. No entanto, referia uma noctúria de 6-8 vezes, urinava mais de 15 vezes durante o dia, usava quatro fraldas durante o dia, só conseguia reter a vontade de urinar num período muito curto (inferior a 3 min.) e que as suas queixas Ihe "roubavam" imensa qualidade de vida. Como antecedentes pessoais destaca-se uma diabetes mellitus tipo 2, hipertensão arterial, hérnias discais lombossagradas, depressão minor e uma intervenção cirúrgica para laqueação das trompas (há mais de 30 anos). Como antecedentes fisiológicos nega consumo de álcool, drogas e tabaco. Realçando alguns dos antecedentes ginecológicos, temos uma G4/P4 (2 partos eutócicos e 2 por cesariana), menopausa há 20 anos e laqueação de trompas há 30 anos. A doente afirmava que já Ihe tinham sido prescritos vários fármacos (anti-muscarínicos) numa 
tentativa de aliviar as queixas de urgência, mas com muito pouco sucesso.

Ao exame objetivo (ginecológico) não foi identificado prolapso dos órgãos pélvicos (POP). Apresentava o stress test, o Q-tip test e a prova de Bonney positivos.

Foi-Ihe pedido um estudo urodinâmico (Fig.s 1 e 2) e uma urocultura. Foi medicada com Betmiga ${ }^{\circledR}$ (mirabegron) 50 mg numa tentativa de alívio sintomático, mas com pouco resultado.

\section{RESULTADO DO ESTUDO URODINÂMICO:}

- Cistometria

\section{RESULTADO DO ESTUDO URODINÂMICO:}

- Relatório do exame

Perante a resposta quase nula à medicação e o resultado do estudo urodinâmico, foi discutida a situação clínica com a doente e foi proposto a realização da seguinte intervenção cirúrgica: - histerectomia supra-cervical e ligamentoplastia com colocação de fita CESA, ambas por via laparoscópica, seguido de colocação de slign sub-uretral (TOT) por via transvaginal (Fig. 3).

Foi explicado todo o procedimento cirúrgico (resultados e possíveis efeitos colaterais), assim como tratar-se de uma técnica nova em fase inicial de implementação em

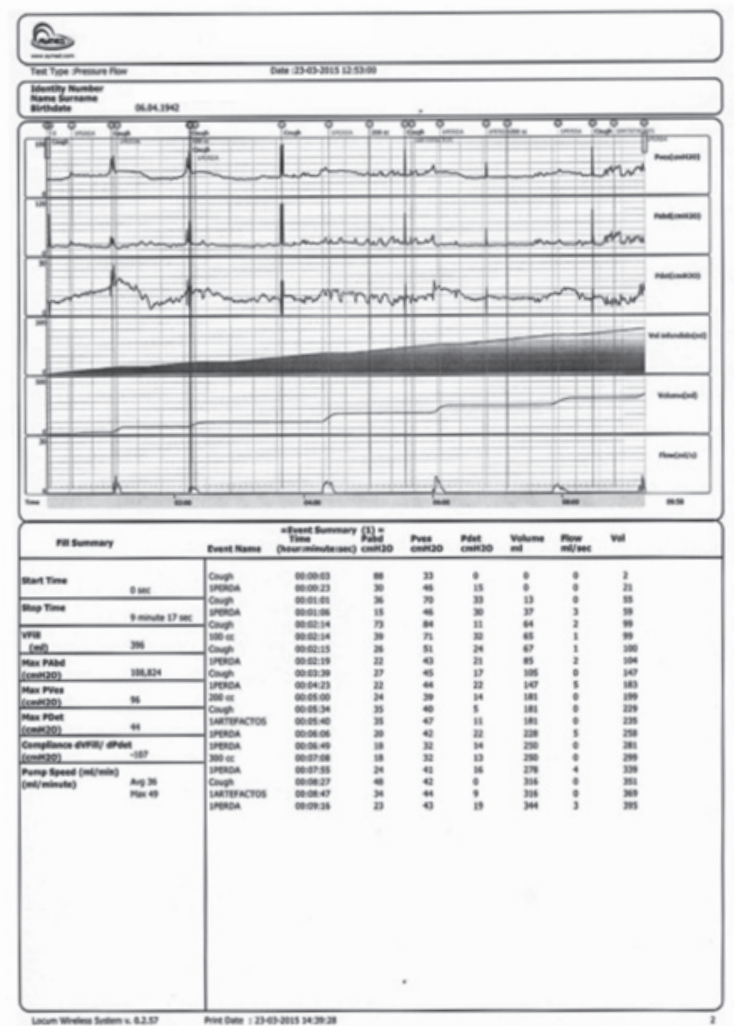

Cistometria
Portugal (24 casos operados à data e com taxa de cura acima dos 85\%), mas já com publicações internacionais e com resultados excelentes (> 82\% de cura) em todos os centros que praticam esta intervenção.

A doente optou por realizar a cirurgia que decorreu no dia 19 de junho de 2015.

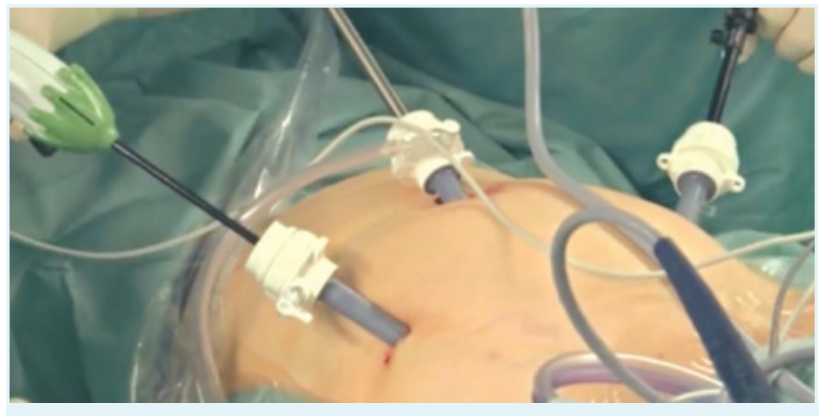

FIGURA 1. Posicionamento dos trocares.

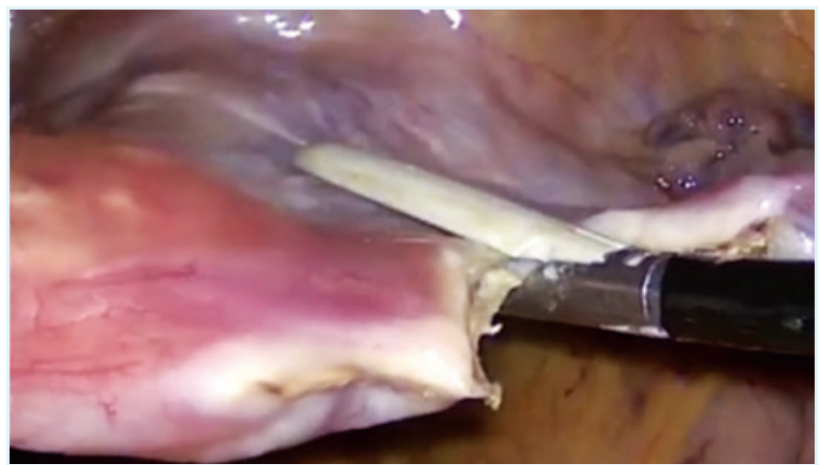

FIGURA 2. Histerectomia supra-cervical.

Relatório de Estudo Urodinâmico Serviço de Urologia

IDENTIFICACÃO:
MÉDICO ASSISTENTE:

DATA: 23-03-2015

UROFUXOMETRL:

Nbo realizada

PERFILOMANOMETRIA URETRAL. - Perfil de Presslo Uretral:

Estudo do regime de pressles ao longo da uretra com sonda manométrica de calibre 8Ch, de 2 vias e perfundidas a $2 \mathrm{~mL} / \min$ revelou: um tónus do mecanismo esfincteriano extermo diminuido

CISTOMANOMETRIA - Estudo da Carra Presalo-Flexo:

"Cistometria de Enchimento e Eswaziamento Vesical"

O estudo do regime de pressibs intravesicais durante o enchimento revelou uma bexiga: a) - Sensibilidude proprioxeptiva aumentada

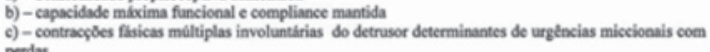
\$) -perdas com as manobras de valsalva. ALPP nato foi possivel realizar por perda imediasta de urina O estudo do regime de pressides intravesicais durante a miceplo revelou que etta tem luear durante uma contraç⿻上丨 flasica do detrusor involunetiria.

Concuusio:

O presente estudo, em contextualizachlo com a clinica, $\boldsymbol{\epsilon}$ sugestive do dingnóstice de

Incontintencia uriniria de efforeo

Detrusor hiperactiva. Incontintincia urindria de urgtncia.

Incontintncia urinaria de tipo mista.

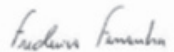

Erederice Fermonha
Urologista

Relatório do exame 


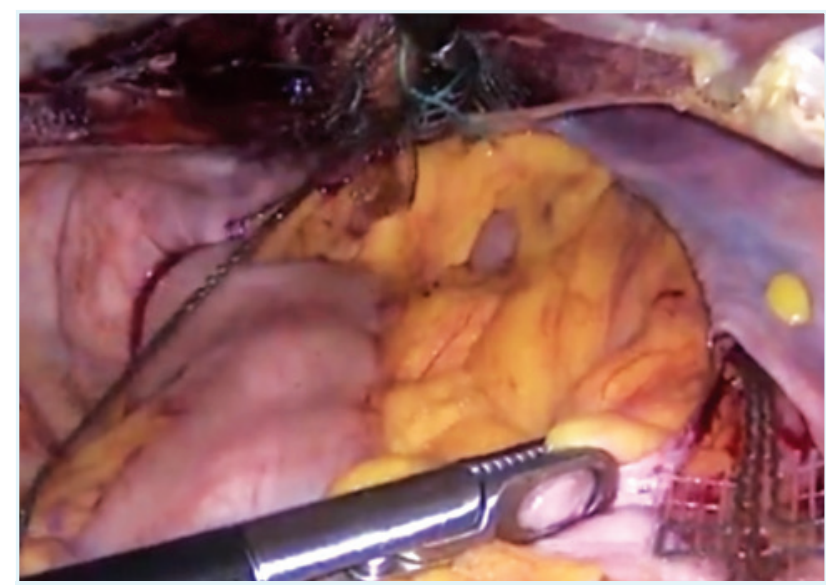

FIGURA 3. Fixação fita CESA ao colo uterino e ao sacro - a nível de S2.

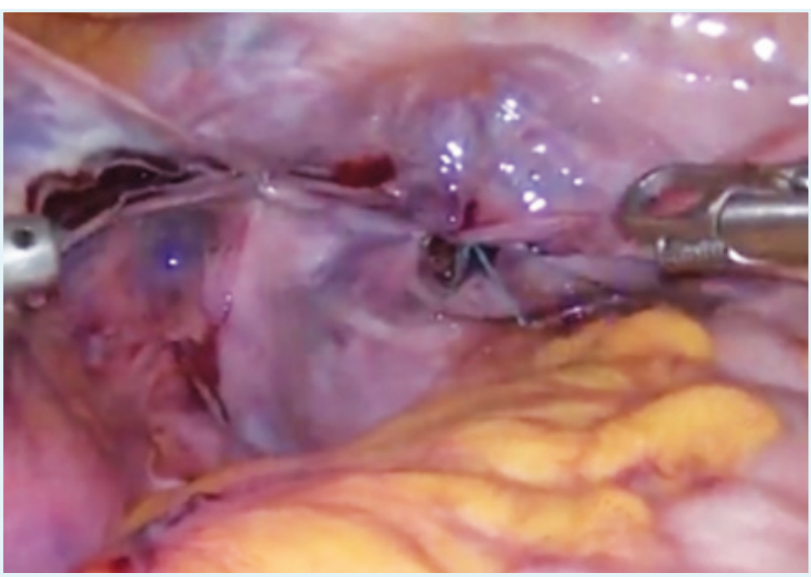

FIGURA 4. Encerramento do retroperitoneu e resultado final.

\begin{tabular}{|c|c|c|c|c|}
\hline Data do convuits $10 / 04 / 2015$ & $\begin{array}{l}\text { antes dis } \\
\text { cinveriata }\end{array}$ & 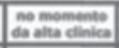 & 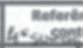 & ciagen \\
\hline 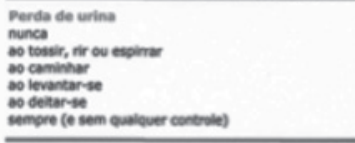 & $\begin{array}{l}0 \\
1 \\
2 \\
3 \\
3 \\
\text { (5) }\end{array}$ & $\begin{array}{l}9 \\
1 \\
2 \\
3 \\
4 \\
5\end{array}$ & $\begin{array}{l}0 \\
1 \\
2 \\
2 \\
3 \\
5 \\
5\end{array}$ & $\begin{array}{l}0 \\
1 \\
2 \\
3 \\
3 \\
5\end{array}$ \\
\hline 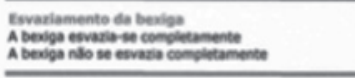 & @ & ๑ & ९ & $\stackrel{0}{2}$ \\
\hline 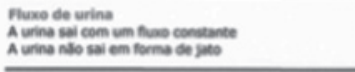 & @ & @ & $\frac{Q}{2}$ & $:$ \\
\hline 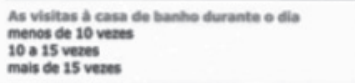 & i & $\underset{2}{\stackrel{1}{2}}$ & $\underset{\frac{1}{2}}{0}$ & $\frac{i}{i}$ \\
\hline 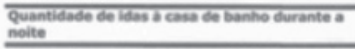 & $6-8 x$ & $2 x$ & $1-2 x$ & $\overline{-}$ \\
\hline 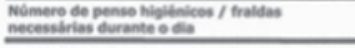 & 4Fialdas & 0 & 0 & - \\
\hline 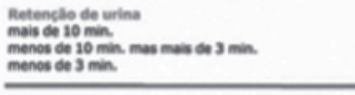 & (3) & $\underset{2}{\stackrel{9}{2}}$ & $\underset{2}{\stackrel{1}{2}}$ & $\begin{array}{l}0 \\
\vdots \\
2\end{array}$ \\
\hline 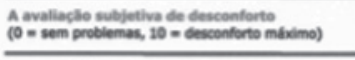 & 10 & 4 & 0 & - \\
\hline 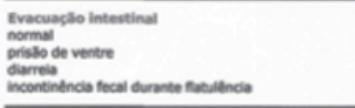 & $\begin{array}{l}0 \\
1 \\
2 \\
3\end{array}$ & $\begin{array}{l}\text { (0) } \\
1 \\
2 \\
3\end{array}$ & $\begin{array}{l}6 \\
1 \\
2 \\
3 \\
3\end{array}$ & $\begin{array}{l}0 \\
1 \\
2 \\
3 \\
\end{array}$ \\
\hline \multicolumn{5}{|c|}{ 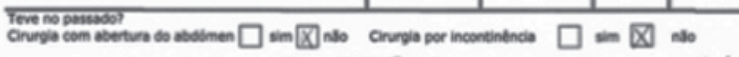 } \\
\hline \multicolumn{5}{|c|}{ 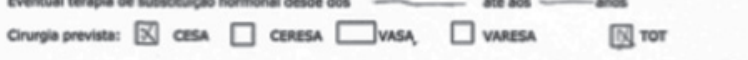 } \\
\hline
\end{tabular}

Comparação da sintomatologia.

\section{INTERVENÇÃO CIRÚRGICA:}

- Histerectomia supra-cervical e ligamentoplastia com substituição dos ligamentos útero-sagrados com aplicação de fita CESA - por via laparoscópica + colocação de sling sub-uretral (TOT) - por via transvaginal

A intervenção cirúrgica e o pós-operatório decorreram sem qualquer intercorrência e a doente teve alta clínica no dia 21 de junho de 2015 (2 dias de internamento).

A sintomatologia da doente foi reavaliada no dia da alta clínica, às quatro e às doze semanas após a intervenção (Fig. 4). A doente afirma - sic: "estou curada, consegui voltar à minha vida normal".

\section{COMPARAÇÃO DA SINTOMATOLOGIA:}

- Antes da cirurgia, no dia da alta clínica e quatro semanas após a intervação

\section{DISCUSSÃO}

Esta técnica está indicada em:

- Todas as mulheres que apresentam sintomas de incontinência urinária de urgência ou mista;

- Exclusão de outras causas possíveis como patologias neurogénicas e congénitas;

- Quando os tratamentos conservadores/médicos não foram eficazes.

A técnica consiste numa abordagem cirúrgica por via abdominal (aberta ou laparoscópica), seguida de histerectomia supra-cervical nos casos de prolapso uterino concomitante. À posterior identificação dos ligamentos útero-sagrados e da vértebra sagrada S2, aplica-se uma prótese (CESANASA) que se fixa ao colo uterino ou à cúpula vaginal, inserida retroperitonealmente e substituindo os, outrora existentes, ligamentos útero-sagrados e é também fixada ao osso sacro (a nível de S2). Quando há prolapso dos órgãos pélvicos associado, nomeadamente uterino ou da cúpula vaginal, esta técnica favorece a correção desse problema ao fortalecer o pavimento pélvico posterior.

Em Portugal já foram realizadas mais de 40 cirurgias com aplicação desta técnica e com resultados - cura cirúrgica - acima dos $85 \%$.

Este caso clínico retrata mais um exemplo do sucesso desta nova técnica cirúrgica.

Podemos afirmar que CESANASA, apesar de ser uma técnica numa fase inicial de desenvolvimento, veio mudar o futuro do tratamento da bexiga hiperativa idiopática. 
CONFLITOS DE INTERESSE: Os autores declaram não ter qualquer conflito de interesse na realização do presente trabalho.

FONTES DE FINANCIAMENTO: Não houve qualquer fonte de financiamento na realização do presente trabalho.

CONFIDENCIALIDADE DOS DADOS: Os autores declaram ter seguido os protocolos da sua instituição acerca da publicação dos dados de doentes.

PROTEÇÃO DE PESSOAS E ANIMAIS: Os autores declaram que os procedimentos seguidos na elaboração do presente trabalho estão em conformidade com as normas das comissões de investigação clínica e de ética, bem como da declaração de Helsínquia e da Associação Médica Mundial.

CONFLICTS OF INTEREST: The authors declare that they have no conflicts of interest.

FINANCIAL SUPPORT: This work has not received any contribution, grant or scholarship.

CONFIDENTIALITY OF DATA: The authors declare that they have followed the protocols of their work center on the publication of data from patients.

PROTECTION OF HUMAN AND ANIMAL SUBJECTS: The authors declare that the procedures followed were in accordance with the regulations of the relevant clinical research ethics committee and with those of the Code of Ethics of the World Medical Association (Declaration of Helsinki).

\section{REFERÊNCIAS}

1. Jäger W, Mirenska $O$, Brügge $S$. Surgical treatment of mixed and urge urinary incontinence in women,Gynecol Obstet Invest. 2012;74:157-64. doi: 10.1159/000339972.

2. Galego P, Oliveira C. CESANASA - Novo tratamento cirúrgico da bexiga hiperactiva., Congresso da Associação Portuguesa de Urologia, 2014. Centro de Congressos do Epic Sana Algarve Hotel, 31 de outubro a 2 de novembro de 2014

3. Rajshekhar S, Mukhopadhyay S, Morris E. Early safety and efficacy outcomes of a novel technique of sacrocolpopexy for the treatment of apical prolapse. Int J Gynaecol Obstet. 2016;135:182-6. doi:10.1016/j.ijgo.2016.05.007.

4. Petros PE, UImsten UI: An integral theory and its method for the diagnosis and management of female urinary incontinence. Sand J Urol Nephrol. 1993;(suppl 153):1-93.

5. Ward KL, Hilton P. A prospective multicenter randomized trial of tension-free vaginal-tape and colposuspension for primary urodynamic stress incontinence: two-year follow-up. Am J Obstet Gynecol. 2004:190:324-31.

6. Shamliyan TA, Kane RL, Wyman J, Wilt TJ. Systematic review: randomized, controlled trials of nonsurgical treatments for urinary incontinence in women. Ann Intern Med 2008;148:45973.

7. DeLancey JO. Structural support of the urethra as it relates to stress urinary incontinence: the hammock hypothesis. Am J Obstet Gynecol. 1994;170:1713-20; discussion 1720-3.

8. Petros PE. An integral theory of female urinary incontinence. Experimental and clinical considerations. Acta Obstet Gynecol Scand Suppl. 1990;153:7-31.

9. Kindermann G, Dimpf T. Kongress der DGGG. Deutsche Gesellschaft für Gynä- kologie und Geburtshilfe 13.-16. Juni 2000, München 2002.

10. Conze J, Junge K, Weiss C, Anurov M, Oettinger A, Klinge U, et al. New polymer for intra-abdominal meshes - PVDF copolymer. J Biomed Mater Res B Appl Biomater. 2008; 87: 321-8. doi: 10.1002/jbm.b.31106.

11. Berger D, Bientzle M. Polyvinylidene fluoride: a suitable mesh material for laparoscopic incisional and parastomal hernia repair! A prospective, observational study with 344 patients. Hernia. 2009;13:167-72. doi: 10.1007/s10029-008-0435-4. 